\section{A program to compute McGraw and Wong's common language effect size indicator}

\author{
WILLIAM P. DUNLAP \\ Tulane University, New Orleans, Louisiana
}

McGraw and Wong (1992) have described a very appealing index of effect size that requires no prior knowledge of statistics to understand, which they termed $C L$, the common language effect size indicator. $C L$ is the probability that a score randomly sampled from one distribution will be larger than a randomly sampled score from a second distribution. McGraw and Wong describe how to compute $C L$ from the means and standard deviations of two groups, using tables of normal curve probability values. The program described herein computes CL without lookup tables but also permits the user to compute $C L$ from Cohen's $d$, from a $t$ test for independent groups, or from point-biserial $r$, the correlation between a dichotomous and a continuous variable. A table giving $C L$ for various values of $d$ is also provided, as are the equations for converting $t$ and $r$ to $d$.

McGraw and Wong (1992) have described an index of effect size that requires only understanding the concept of a probability, but no prior knowledge of statistics, which they termed the common language effect size indicator $(C L)$. McGraw and Wong asked the reader to consider randomly selecting a young adult male and a young adult female; their $C L$ index is the probability that a measure made on the male will exceed that made on the female. If the measure was standing height, McGraw and Wong show that this probability is about .92 . If, on the other hand, there were no difference between the mean heights of males and females, this probability, the $C L$, would equal .5 , an equal likelihood of males' height exceeding that of females. Despite the appealing simplicity of $C L$, a literature review by Kirk (1996) reported that, of 266 reported effect sizes in 391 articles surveyed, not a single one used $C L$; perhaps researchers are hesitant to compute the index, even with the simple instructions given by McGraw and Wong.

The way $C L$ is computed is to consider the distribution of all the possible differences between pairs of points from the two groups-in the above example, subtracting female heights from male heights. This distribution has a known mean and standard error, and, assuming normality, one can compute the ratio of the mean difference to the standard error of differences, then look up the probability that this value will not be exceeded from tables of the normal curve, which is the value of $C L$.

Unfortunately, McGraw and Wong (1992) only showed how to compute $C L$ from means and standard deviations.

Correspondence concerning this article should be addressed to W. P. Dunlap, Department of Psychology, Tulane University, New Orleans, LA 70118 (e-mail: dunlap@mailhost.tcs.tulane.edu).
The present article gives the relationship between $C L$ and Cohen's $d$. Because $d$ can be computed from means and standard deviations, from a $t$ test for independent groups, or from the point-biserial correlation relating a dichotomous grouping factor to a continuous variable, the relationship between $C L$ and $d$ serves as a common path to compute $C L$ from a number of possible starting points. Therefore, the purpose of this article is to describe the needed relationships, to provide a table giving $C L$ from values of $d$ (up to $d=1.99$ ), and to provide an interactive computer program that will compute $C L$ from a number of different starting points.

\section{Necessary Relationships}

In order to compute $d$ from means and standard deviations, one first must estimate the common standard deviation by

$$
s d=\sqrt{\frac{\left(n_{1}-1\right) s d_{1}^{2}+\left(n_{2}-1\right) s d_{2}^{2}}{n_{1}+n_{2}-2}},
$$

where $s d_{i}$ are the standard deviations and $n_{i}$ are the corresponding sample sizes (Hedges, 1981). When the sample sizes are equal, this equation simplifies to

$$
s d=\sqrt{\frac{s d_{1}^{2}+s d_{2}^{2}}{2}}
$$

the square root of the average variance. Cohen's $d$ is equal to

$$
d=\frac{M_{1}-M_{2}}{s d},
$$

where $M_{1}$ and $M_{2}$ are the two group means. From $d$, the value of $C L$ can be computed by finding the integral of the normal distribution from negative infinity to $d / \sqrt{2}$ (which equals $0.7071 d$ ). The values of $C L$ from corresponding values of $d$ are given in Table 1. For example, if $d=0.73$, find the row labeled .7 and the column headed by .03 , and where they intersect we find $C L=.697$. If the first mean is smaller than the second, so that $d$ is negative, look up the absolute value of $d$ in Table 1 but subtract the corresponding $C L$ value from one, getting a value for $C L$ that is less than .5 .

Now, to compute $C L$ from various other starting points, we need the following relationships:

$$
d=t \sqrt{\frac{n_{1}+n_{2}}{n_{1} n_{2}}}
$$

and

$$
d=r \frac{\sqrt{\frac{\left(n_{1}+n_{2}-2\right)\left(n_{1}+n_{2}\right)}{n_{1} n_{2}}}}{\sqrt{1-r^{2}}}
$$


Table 1

Value of the Common Language Effect Size Indicator, $C L$, for Corresponding Values of Cohen's $d(0.00-1.99)$

\begin{tabular}{ccccccccccc}
\hline$d$ & .00 & .01 & .02 & .03 & .04 & .05 & .06 & .07 & .08 & .09 \\
\hline .00 & .500 & .503 & .506 & .508 & .511 & .514 & .517 & .520 & .523 & .525 \\
.10 & .528 & .531 & .534 & .537 & .539 & .542 & .545 & .548 & .551 & .553 \\
.20 & .556 & .559 & .562 & .565 & .567 & .570 & .573 & .576 & .578 & .581 \\
.30 & .584 & .587 & .590 & .592 & .595 & .598 & .600 & .603 & .606 & .609 \\
.40 & .611 & .614 & .617 & .619 & .622 & .625 & .628 & .630 & .633 & .636 \\
.50 & .638 & .641 & .643 & .646 & .649 & .651 & .654 & .657 & .659 & .662 \\
.60 & .664 & .667 & .669 & .672 & .675 & .677 & .680 & .682 & .685 & .687 \\
.70 & .690 & .692 & .695 & .697 & .700 & .702 & .705 & .707 & .709 & .712 \\
.80 & .714 & .717 & .719 & .721 & .724 & .726 & .728 & .731 & .733 & .735 \\
.90 & .738 & .740 & .742 & .745 & .747 & .749 & .751 & .754 & .756 & .758 \\
1.00 & .760 & .762 & .765 & .767 & .769 & .771 & .773 & .775 & .777 & .780 \\
1.10 & .782 & .784 & .786 & .788 & .790 & .792 & .794 & .796 & .798 & .800 \\
1.20 & .802 & .804 & .806 & .808 & .810 & .812 & .814 & .815 & .817 & .819 \\
1.30 & .821 & .823 & .825 & .827 & .828 & .830 & .832 & .834 & .835 & .837 \\
1.40 & .839 & .841 & .842 & .844 & .846 & .847 & .849 & .851 & .852 & .854 \\
1.50 & .856 & .857 & .859 & .860 & .862 & .863 & .865 & .867 & .868 & .870 \\
1.60 & .871 & .873 & .874 & .875 & .877 & .878 & .880 & .881 & .883 & .884 \\
1.70 & .885 & .887 & .888 & .889 & .891 & .892 & .893 & .895 & .896 & .897 \\
1.80 & .898 & .900 & .901 & .902 & .903 & .905 & .906 & .907 & .908 & .909 \\
1.90 & .910 & .912 & .913 & .914 & .915 & .916 & .917 & .918 & .919 & .920 \\
\hline
\end{tabular}

where $t$ is the $t$ test for independent groups and $r$ is the point-biserial correlation between a dichotomous variable indicating group membership and the dependent variable. When the sample sizes of the two groups are equal $\left(n_{1}=\right.$ $n_{2}=n$ ), the equations simplify to

$$
d=t \frac{2}{n}
$$

and

$$
d=2 r \frac{\frac{n-1}{n}}{1-r^{2}} .
$$

Therefore, one can make the conversion to $d$ by using the above equations and then look up $d$ in Table 1, to get the conversion to $C L$.

\section{The Program}

An interactive program, described in the Appendix, was written in FORTRAN and uses a function described by Dunlap and Duffy (1975) to compute integrals of the normal distribution, in order to compute $C L$ from various different types of input. The user may choose to input means and standard deviations, $d, t$ for independent groups, or the point-biserial $r$. Values of $d$ and $C L$ are returned. This program avoids any hand calculations or table lookup. The FORTRAN statements used are very simple and primitive, so that the program should compile on virtually any available FORTRAN compiler. Another option is to send a DOS formatted disk to the author, which will be returned with a copy of an executable program that will run under DOS on an IBM-compatible PC.

\section{Discussion}

The $C L$ requires only that one understand the concept of a probability. Various other effect size measures, such as Cohen's $d$ (Cohen, 1977), Rosenthal and Rubin's (1982) binomial effect size, $r^{2}$ (the variance explained), and many others all require, to a greater or a lesser extent, some understanding of statistics. The $C L$ index, on the other hand, permits quite simple statements, such as "if a randomly selected adult male is compared with a randomly selected adult female, the chance that the male is taller is 93\%"; this is a statement that is as easily understood by a person not trained in science or statistics as it is by a well-trained scientist. Increasingly we are urged in behavioral research to go beyond simple reports of significance to provide estimates of effect size (e.g., Kirk, 1996). The problem is that not only is there a bewildering array of such measures, but almost all require an understanding of correlation, variance, or various other statistical concepts. The $C L$ index, using either the table provided in this article or the interactive program described, is relatively easy to compute and provides an unambiguous statement of effect size.

Statistical issues. Although no deep understanding of statistics is required to interpret and understand the intent of $C L$ as an index of effect size, statistical assumptions are relevant to its accuracy, and certain simplifications may lead to bias. Clearly, $C L$ must be estimated from sample information; therefore, it provides, at best, an estimate of the population probability. When, for example, individual sample sizes are not known but are assumed to be equal, $C L$ can be shown to underestimate the value that would have been calculated if actual unequal sample sizes were known and used. Sometimes actual sample 
sizes can be judged from the experimental description, but at other times, more global information, such as degrees of freedom, may require that they be inferred, in which case equality is often assumed. $C L$ also depends on the assumption of normality, the most common deviation from which is skew. Skew generally leads to loss of power and lower values of the $t$ test; therefore, it will tend to yield conservative values of $C L$ (see Levine \& Dunlap, 1982). When estimates of the standard deviation are pooled (see Equations 1 and 2) for purposes of computing $d$ or $C L$, one has assumed homogeneity of population variances. If one assumes equal variance when, in fact, the population variances are unequal, $C L$ will be overestimated, and when combined with unequal sample sizes, these distortions can be great. In this case, it is necessary to compute the standard error of the difference scores directly from the estimated standard deviations of the different groups by taking the square root of

$$
s d_{\mathrm{A}-\mathrm{B}}^{2}=s d_{\mathrm{A}}^{2}+s d_{\mathrm{B}}^{2} \text {. }
$$

Finally, it is possible to compute $C L$ from the results of experimental designs with matched observations or repeated measures, as McGraw and Wong (1992) pointed out. One must be very careful, however, not to compute $C L$ from the $t$ test for such designs without making the necessary adjustments and taking the precautions described by Dunlap, Cortina, Vaslow, and Burke (1996).

To better understand the value of the simplicity of $C L$, let us examine a case of a culturally biased test in applied psychology. Let us suppose that the point-biserial correlation between the dichotomous variable coding race (black $=1$, white $=2$ ) and an ability test score was .3 , which would indicate that, on average, whites would receive somewhat higher scores than blacks on the test.
There are many, however, who would point out that the relationship is a weak one, because race only explains $9 \%$ of the variance. Many of the effect size measures are in the form of variance explained, but who really understands what variance explained means? If we assume the correlation was based on samples of 50 blacks and 50 whites, we can use Equation 7 to compute a value of $d$ equal to 0.62 . Looking this value up in Table 1 gives $C L$ equal to .67 . This means that, if a black and a white were contending for the same job and if the biased ability test were used to decide who got it, $67 \%$ of the time, the white would be chosen because of the bias. This is a much more concrete statement than a vague reference to $9 \%$ explained variance.

\section{REFERENCES}

CoHEN, J. (1977). Statistical power analysis for the behavioral sciences (2nd ed.). Hillsdale, NJ: Erlbaum.

Dunlap, W. P., Cortina, J. M., Vaslow, J. B., \& Burke, M. J. (1996). Meta-analysis of experiments with matched groups or repeated measures designs. Psychological Methods, 1, 170-177.

DUNLAP, W. P., \& DUFFY, J. A. (1975). FORTRAN IV functions for calculating exact probabilities associated with $z, \chi^{2}, t$, and $F$ values. $B e$ havior Research Methods \& Instrumentation, 7, 59-60.

HEDGEs, L. V. (1981). Distributional theory for Glass's estimator of effect size and related estimators. Journal of Educational Statistics, 6 , 107-128.

KIRK, R. E. (1996). Practical significance: A concept whose time has come. Educational \& Psychological Measurement, 56, 746-759.

Levine, D. W., \& Dunlap, W. P. (1982). Power of the F-test with skewed data: Should one transform or not? Psychological Bulletin, 92, 272-280.

McGraw, K. O., \& Wong, S. P. (1992). A common language effect size statistic. Psychological Bulletin, 111, 361-365.

Rosenthal, R., \& RuBin, D. B. (1982). A simple, general purpose display of magnitude of experimental effect. Journal of Educational Psychology, 74, 166-169. 


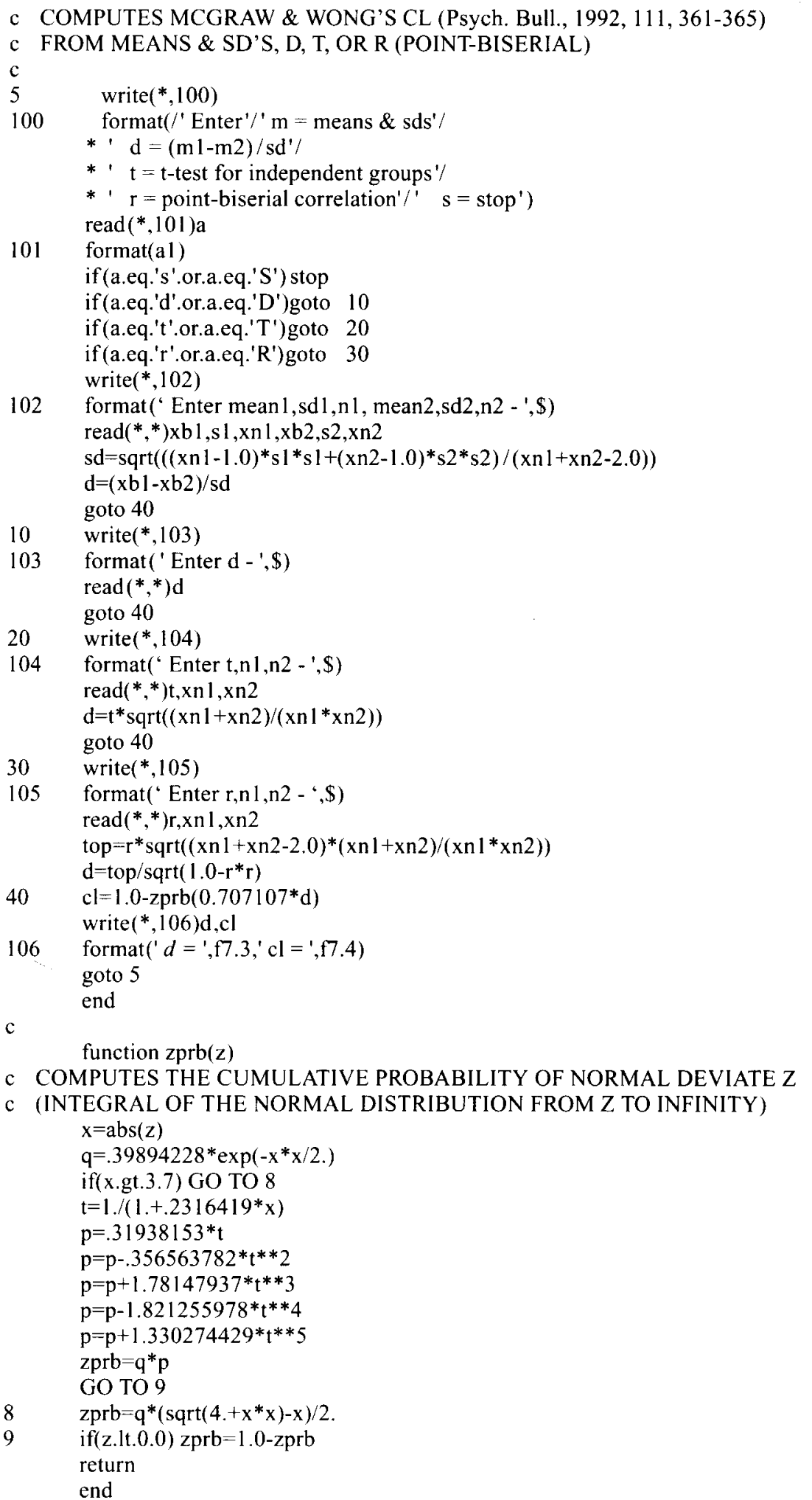

